\title{
Improved reconstructions of random media using dilation and erosion processes
}

\author{
Chase E. Zachary ${ }^{1}$ and Salvatore Torquato ${ }^{1,2,3,4,5 *}$ \\ ${ }^{1}$ Department of Chemistry, ${ }^{2}$ Department of Physics, \\ ${ }^{3}$ Princeton Center for Theoretical Science, \\ ${ }^{4}$ Program in Applied and Computational Mathematics, \\ and ${ }^{5}$ Princeton Institute for the Science and Technology of Materials, \\ Princeton University, Princeton, New Jersey 08544, USA
}

\begin{abstract}
By using the most sensitive two-point correlation functions introduced to date, we reconstruct the microstructures of two-phase random media with heretofore unattained accuracy. Such media arise in a host of contexts, including porous and composite media, ecological structures, biological media, and astrophysical structures. The aforementioned correlation functions are special cases of the so-called canonical n-point correlation function $H_{n}$ and generalize the ones that have been recently employed to advance our ability to reconstruct complex microstructures [Y. Jiao, F. H. Stillinger, and S. Torquato, Proc. Nat. Acad. Sci. 106, 17634 (2009)]. The use of these generalized correlation functions is tantamount to dilating or eroding a reference phase of the target medium and incorporating the additional topological information of the modified media, thereby providing more accurate reconstructions of percolating, filamentary, and other topologically complex microstructures. We apply our methods to a multiply-connected "donut" medium and a dilute distribution of "cracks" (a set of essentially zero measure) demonstrating improved accuracy in both cases with implications for higher-dimensional and biconnected two-phase systems. The high information content of the generalized two-point correlation functions suggests that it would be profitable to explore their use to characterize the structural and physical properties of not only random media, but also molecular systems, including structural glasses.
\end{abstract}

\footnotetext{
*torquato@princeton.edu
} 


\section{INTRODUCTION}

Two-phase random media, which are partitions of space into two distinct phases with interfaces that are known only probabilistically, abound in a host of situations, including composite materials [1 5], cosmological [6, 7] and geophysical structures [1], and biological systems [8, 9]. Importantly, the effective physical and chemical properties of random media are rigorously related to microstructural correlations functions [1, 2]. In principle, complete microstructural information requires an enormously large set of such correlation functions, including statistical descriptors describing higher-order correlations (e.g., three-point, fourpoint, and higher-order statistics). Since in any practical setting only lower-order correlation functions are available, it is of paramount interest to quantify the amount of microstructural information contained in such reduced statistics and then to identify the most sensitive descriptors.

Microstructure reconstruction techniques provide a means of probing these issues. Reconstruction of a random medium that matches limited structural information, i.e., finite set of targeted lower-order correlation functions of the system, is an intriguing inverse problem [10, 11]. By comparing unconstrained correlation functions between the original and reconstructed medium, one can quantify the capacity of the reduced statistics to specify the important structural features of the medium [12]. An effective reconstruction procedure enables one to generate accurate renditions of random media at will, and subsequent analysis can be performed on the reconstruction to obtain desired macroscopic properties nondestructively [1, 13].

Although there has been significant progress on reconstruction algorithms [1, 14 16], it remains a challenge to reconstruct accurately percolating microstructures, sets of essentially zero measure (e.g., cracked media and filamentary structures), and multiply connected microstructures. In this paper, we introduce a procedure that enables one to begin to reconstruct such microstructures with heretofore unattained accuracy.

It is noteworthy that a panoply of different types of correlation functions arise in rigorous theories of structure-property relations for heterogeneous media, which includes the $n$-point probability function $S_{n}, n$-point surface correlation functions, pore-size functions, lineal-path functions, nearest-neighbor functions, among others [1]. It was shown that all of these statistical descriptors are special cases of the more general canonical n-point cor- 
relation function $H_{n}$, which is a hybrid correlation-density function and can be represented analytically for a wide class of microstructures [1, 17]. Indeed, $H_{n}$ characterizes a modified random medium that involves uniformly "dilating" or "eroding" a reference phase in the normal direction to the two-phase interface in the actual random medium [1]. Dilation is achieved by placing a "test" sphere of radius $\delta$ into the non-reference phase and determining the space available to the sphere as if the reference phase was impenetrable to the test sphere. The space unavailable to the test sphere is considered to be the modified or dilated reference phase. The erosion process simply reverses this procedure such that the reference phase is allowed to be penetrated by a distance $\delta$ normal to the two-phase interface. The quantity $H_{n}$ is an even more general quantity since it allows for multiple test spheres, each of which has a different size; see Refs. [1] and [17] for details. Thus, $H_{n}$ contains considerably more information than any of the types of correlations functions that arise in the theory of heterogeneous media. We will use special cases of $H_{2}$ in our reconstruction method, as described below.

In practice, the enormous information content of the full $n$-point canonical function $H_{n}$ is neither experimentally nor theoretically accessible, and one must instead rely on reduced statistical information about the microstructure. It is an open and fundamental problem to identify via reconstruction techniques lower-order statistical descriptors that can be both manageably measured and yet reflect a substantial portion of the complete microstructural information contained in $H_{n}$. The most prominent example of reduced statistical information in the two-point probability function $S_{2}(\mathbf{r})$, probability of finding two points separated by the displacement vector $\mathbf{r}$ in one of the phases. This function is accessible from scattering experiments [18] or tomography [19]. Such information alone has been shown to be generally insufficient to render accurate reconstructions [11, 12].

The most successful reconstruction procedures to date have directly incorporated connectedness information about the target media. In particular, Jiao, Stillinger and Torquato [15] have shown that incorporation of the two-point cluster function $C_{2}$, experimentally obtainable from tomography or other three-dimensional imaging techniques [1], results in superior reconstructions. The two-point cluster function defines the probability of finding two points in both the same phase and in the same connected cluster of the microstructure [1, 20]. The function $C_{2}(\mathbf{r})$ is the connectedness contribution to the two-point probability function, namely, $S_{2}(\mathbf{r})=C_{2}(\mathbf{r})+E_{2}(\mathbf{r})$, where $E_{2}$ is the two-point blocking function, cor- 
responding to the case where two points fall within different clusters of the same phase. It has been shown that $C_{2}$ contains substantially more information in excess to $S_{2}$ than even the three-point probability function $S_{3}$, thereby highlighting the inefficiency of incorporating additional information via the usual higher-order correlation functions [15].

However, there remains a large class of microstructures that are difficult to characterize accurately using only $S_{2}$ and $C_{2}$. Examples of such systems include heterogeneous media with percolating and/or multi-connected phases [21], systems with zero-measure structures such as cracked materials and filamentary structures that arise in the large-scale structure of the Universe [22], and other topologically complex media. In particular, $C_{2}$ works best when the phase of interest is below its percolation threshold; otherwise, it is a long-ranged function that does not reflect structural details. Moreover, when the phases occupy effectively a space of Lebesgue measure zero, the probability of finding a point "within" the set identically vanishes; these elements are therefore unable to contribute to either $S_{2}$ or $C_{2}$.

In this paper, we present an improved reconstruction procedure that naturally generalizes the $S_{2}-C_{2}$ methodology presented in Ref. [15]. Fundamental to our work is the information contained in the void space external to the phase of interest [23, 24]. In particular, we incorporate special and new cases of $\mathrm{H}_{2}$, which contain additional topological information associated with modifications of the target microstructure via "dilation" and "erosion" processes, thereby allowing a more accurate reconstruction procedure. Importantly, our methods contain previously-reported results for $S_{2}$ and $C_{2}$ as a special case, thereby providing a general means to study the amount of information contained in lower-order correlation functions.

\section{GENERALIZED TWO-POINT CORRELATION FUNCTIONS: SPECIAL CASES $\mathrm{OF} \mathrm{H}_{2}$}

Previous studies on reconstructions of heterogeneous media [15] have utilized the pore-size probability function $F(\delta)$, defined as the probability of finding a spherical cavity of radius $\delta$ contained in the void space external to the phase of interest. The pore-size probability function contains short-range connectedness information within the void space and can also be interpreted as the volume fraction of the pore space after uniformly dilating the reference phase by a linear scale $\delta$ [23]; note that erosion by a linear scale $\delta$ corresponds to the 
continuous extension of $F$ to negative values of $\delta$. As such, it is a sensitive one-point descriptor of heterogeneous media implicitly containing limited higher-order information.

Higher-order pore-size probability functions are simply special cases of the canonical $n$-point correlation function $H_{n}$. In particular, the $n$-point pore-size probability function $F_{n}\left(\mathbf{r}^{n} ;\{\delta\}_{n}\right)$ is the probability of finding $n$ spherical cavities of radii $\delta_{1}, \ldots, \delta_{n}$, all within

the "void" space external to the reference phase; in the notation of Ref. [17], $F_{n}\left(\mathbf{r}^{n} ;\{\delta\}_{n}\right) \equiv$ $H_{n}\left(\mathbf{r}^{n} ; \varnothing ; \varnothing\right)$. Of particular interest here is the two-point pore-size probability function $F_{2}(\mathbf{r} ; \delta)$ for a statistically homogeneous medium, where $\mathbf{r}=\mathbf{r}_{2}-\mathbf{r}_{1}$ and $\delta=\delta_{1}=\delta_{2}$. This function also contains short-range connectedness information about the modified void space and can be interpreted as the two-point probability function $S_{2}$ for the void space after uniformly dilating the reference phase by a linear scale $\delta$. As such, it can be decomposed as $F_{2}(\mathbf{r} ; \delta)=K_{2}(\mathbf{r} ; \delta)+M_{2}(\mathbf{r} ; \delta)$, where $K_{2}$ and $M_{2}$ are the two-point pore-size cluster and blocking functions, respectively.

Since $F_{2}(\mathbf{r} ; \delta) \rightarrow S_{2}(\mathbf{r})$ (for the void space) as $\delta \rightarrow 0$, it follows that $F_{2}$ and its cluster counterpart $K_{2}$ rigorously contain more information about the microstructure than the standard $S_{2}$ and $C_{2}$ functions alone. Thus, we incorporate these generalized $S_{2}$ and $C_{2}$ functions in reconstructions, which amounts to applying dilation or erosion processes to modify the phase connectedness of a target microstructure. We then perform a reconstruction procedure on the modified medium, which is expected to yield improved reconstructions of the original unmodified medium.

\section{RECONSTRUCTION PROCEDURE AND APPLICATIONS}

Our reconstruction procedure is a modification of the Jiao-Stillinger-Torquato methodology [15], which extends the Yeong-Torquato simulated annealing algorithm [11] to include clustering information. In particular, we overlay the image with a set of $N^{d}$ pixels taking values 1 (within the phase of interest) or 0 (in the void space), where $d$ is the Euclidean dimension. By measuring the distances between void and surface pixels, we perform an initial dilation or erosion process to modify the microstructure prior to reconstruction. Reconstruction of the modified structure then proceeds by stochastic optimization of the following 


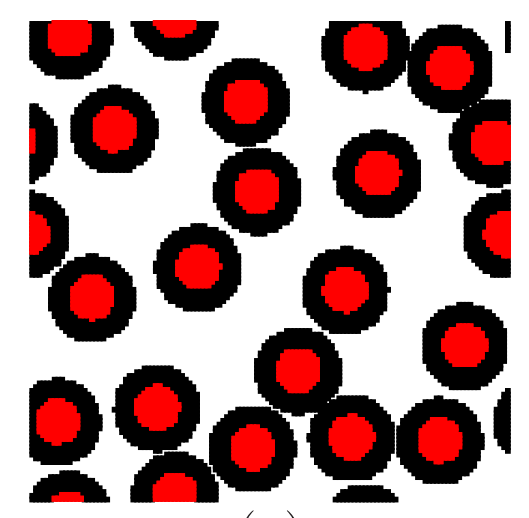

(a)

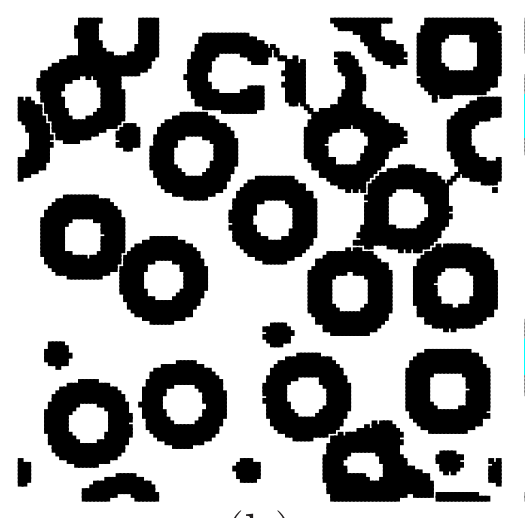

(b)

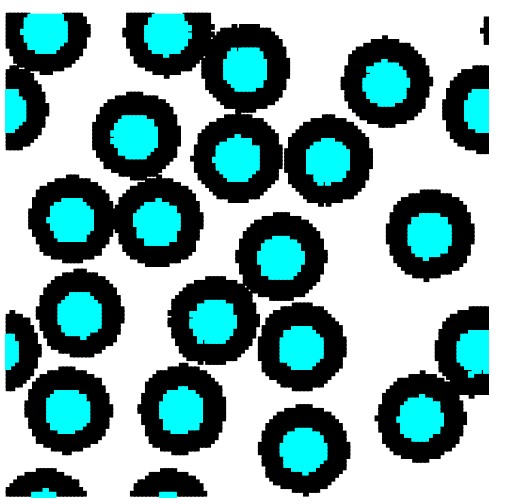

(c)

FIG. 1. (Color online) The doubly-connected "donut" medium. (a) Original microstructure (outer rings) with modified target structure (inner disks; red online) after an erosion process. (b) Direct $S_{2}(r)-C_{2}(r)$ reconstruction of the original microstructure. (c) $S_{2}(r)-C_{2}(r)$ reconstruction of the modified target structure (inner disks; blue online) along with the final reconstructed system (outer rings) after a dilation process. All reconstructions have a final energy (error) at most $E \sim 10^{-8}$.

objective (energy) function [1, 11]:

$$
E=\sum_{\alpha=1}^{M} \sum_{j=1}^{S}\left[f_{j}\left(\mathbf{r}_{\alpha}\right)-f_{j}^{\prime}\left(\mathbf{r}_{\alpha}\right)\right]^{2},
$$

where $M$ is the number of sampling points and $S$ is the number of constrained correlation functions $f_{j}$ with target values $f_{j}^{\prime}$. In this work we consider radially-averaged functions [e.g., $S_{2}(r)$ ], which are more readily accessible from scattering experiments and more relevant to studies in the thermodynamic limit [12]. However, our methods can be directly extended to include anisotropic correlation functions. The Jiao-Stillinger-Torquato procedure tracks the occurrence of "cluster" and "surface" events to update efficiently the cluster and boundary lists at each step of the optimization procedure [15]. A cooling schedule is chosen such that the energy $E$ approaches its ground-state value within a very small tolerance [25].

We illustrate our improved reconstruction methodology by studying the doubly-connected "donut" structure shown in Figure 1, consisting of annuli distributed in the plane. It is noteworthy that nanoparticles of mesoporous silica shells with hollow interiors have been designed with similar cross-sectional structures for applications to biomedical imaging and targeted drug delivery [26]. The uniformity of the nanoparticle sizes and shapes, including the hollow interiors, is particularly important and can be directly quantified using a combination of imaging techniques and pore-size distribution functions [26]. 


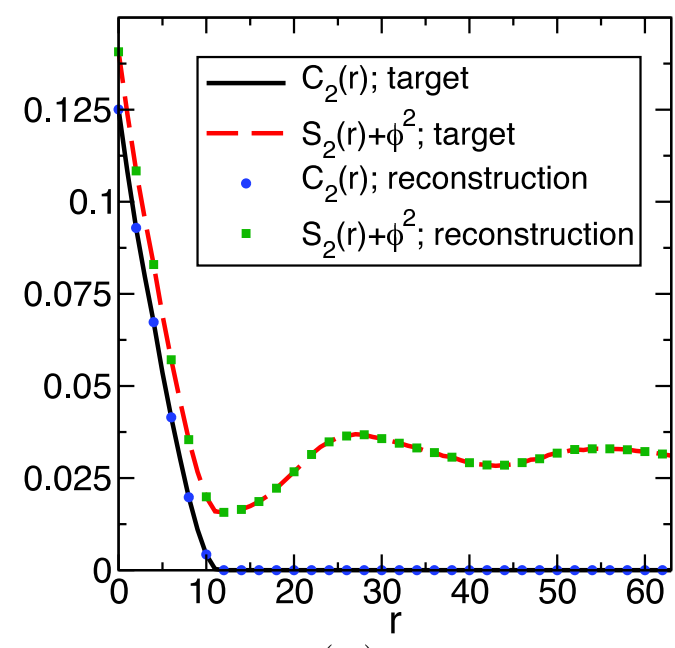

(a)

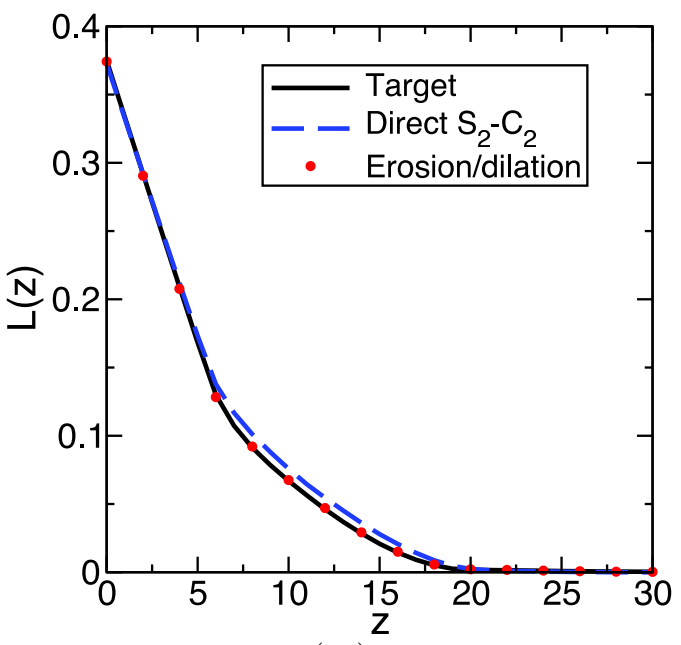

(b)

FIG. 2. (Color online) (a) $S_{2}(r)$ and $C_{2}(r)$ for the modified target disk structure following an erosion process and for the reconstructed disk microstructure. (b) Lineal path functions $L(z)$ for the original target donut structure, a direct $S_{2}(r)-C_{2}(r)$ reconstruction of the donut system, and the reconstructed material using erosion and dilation processes.

The connectedness information of the donut structures is nontrivial because the twodimensional annulus is not a simply-connected topological object. A direct reconstruction using $S_{2}$ and $C_{2}$ shows that, while the two-point cluster function is able to identify situations where points fall within the same cluster, it cannot distinguish situations where the "donut shells" are closed and open. Furthermore, isolated disks of particles appear in the reconstructed image with diameter approximately equal to the length over which $C_{2}$ is nonzero.

We are able to improve the reconstruction procedure by targeting the spatial information contained in the "cores" of the ring structures. Although the annuli surrounding the cores are not simply-connected, by performing an erosion process on the initial microstructure, we generate a "modified" system composed of simply-connected, spatially separated disks in the plane (see Figure 1). Reconstruction of the modified medium using $S_{2}$ and $C_{2}$ is able to reproduce the statistical distribution of the particle centers, and after applying a subsequent dilation process, the original microstructure is recovered with greater fidelity (Figures 1 and 2).

We quantify the accuracy of the reconstruction procedure by measuring some unconstrained correlation function not targeted by the reconstruction algorithm. In Figure 2, we 
compare the lineal path function $L(z)$, defined as the probability of finding a connected linear segment of length $z$ contained completely in the reference phase, for the original donut structure, the direct $S_{2}-C_{2}$ reconstruction, and our reconstruction using erosion and dilation processes. The direct reconstruction algorithm is not able to capture accurately this type of short-range continuous connectedness information, accounting for the poorer reconstruction in Figure 1. However, the lineal path functions of the original system and the reconstructed material utilizing erosion and dilation processes match closely, implying that this information is already implicitly encoded in the spatial distribution of the donut centers. By utilizing effective two-point correlation functions that directly probe the cores of the donut inclusions, we are able to reduce the topological complexity associated with the reconstruction procedure without losing fundamental information about the connectedness properties of the microstructure.

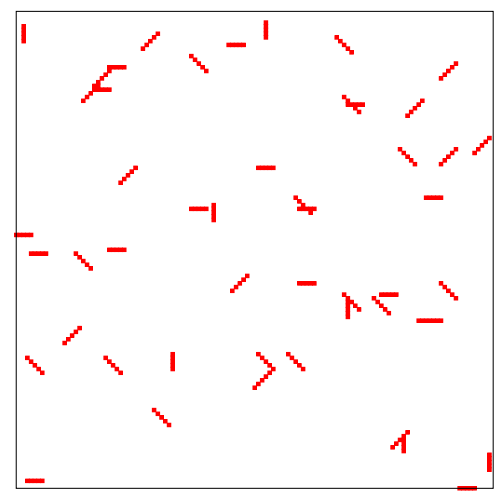

(a)

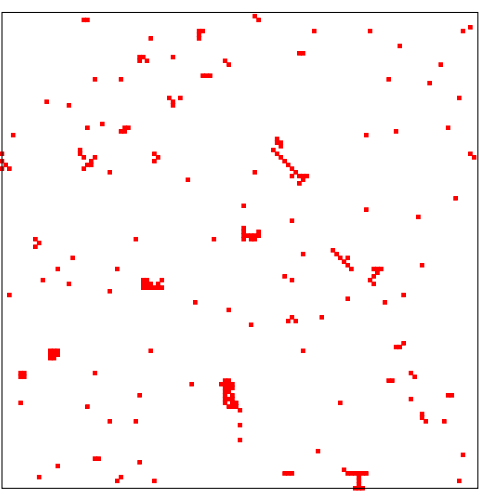

(b)

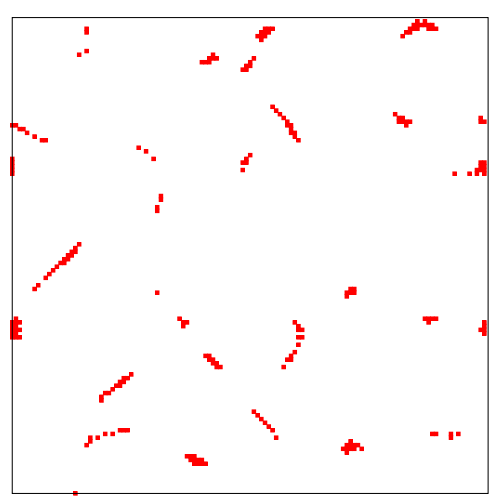

(c)

FIG. 3. (Color online) (a) Target microstructure consisting of a dilute distribution of cracks of nearly-zero measure. (b) Direct $S_{2}(r)-C_{2}(r)$ reconstruction of the target cracked structure. (c) $S_{2}(r)-C_{2}(r)$ reconstruction of the dilated target microstructure followed by an erosion process. All reconstructions have a final energy (error) at most $E \sim 10^{-8}$.

We have also applied our methodology to the reconstruction of a dilute distribution of "cracks" of nearly-zero measure, shown in Figure 3. Note that this prototypical cracked material is very difficult to reconstruct directly, even utilizing the two-point cluster function (see Figure 3b). Although the cracks are given a finite size by the pixelation procedure, the volume fraction associated with the cracks is small, and a direct $S_{2}-C_{2}$ reconstruction preserves neither the cracked elements of the target structure nor the void-space distribution.

However, by dilating the target structure and reconstructing the dilated system, we have 


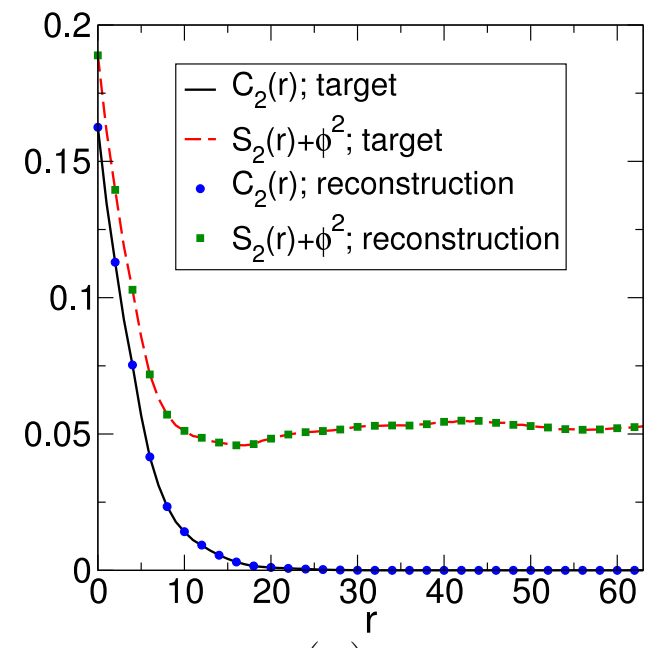

(a)

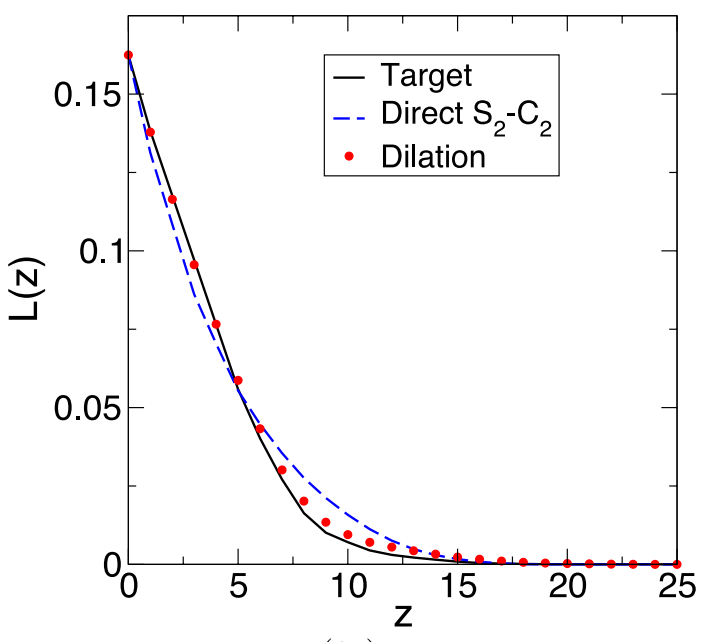

(b)

FIG. 4. (Color online) (a) $S_{2}(r)$ and $C_{2}(r)$ for the dilated target cracked structure and its reconstruction. (b) Lineal path functions for the dilated target cracked structure, its reconstruction, and the dilation of the direct $S_{2}(r)-C_{2}(r)$ reconstruction of the initial target microstructure.

been able to improve the accuracy of the reconstruction procedure. Figure 4 shows excellent agreement between the two-point probability and cluster functions for the dilated target and reconstructed microstructures, and calculation of the lineal path functions demonstrates that the dilation methodology provides quantitatively substantial improvement in the accuracy of the reconstruction. As previously mentioned, extended filamentary structures arise in studies of the large-scale structure of the Universe [6, 27], and our results therefore suggest that twopoint pore-size correlation functions contain important structural information about such systems, beyond even the standard two-point probability function $S_{2}$ and the two-point cluster function $C_{2}$.

\section{DISCUSSION AND CONCLUSIONS}

We have introduced a different reconstruction procedure that utilizes two-point pore-size correlation functions embodied in $\mathrm{H}_{2}$ in conjunction with clustering information to improve reconstructions of two-phase random heterogeneous media. It is noteworthy that these twopoint functions and the canonical $n$-point function $H_{n}$ are generalizations of the Minkowski functionals [28]. Our results support the important notion that the void space is fundamental to the microstructural properties of a heterogeneous system [23, 24]. Our methodology 
provides a natural extension of the Jiao-Stillinger-Torquato reconstruction algorithm and the correlation functions used therein [15]. A major conclusion of this earlier study was that the two-point cluster function $C_{2}$ employed actually embodies higher-order structural information in a way that makes it a highly sensitive statistical descriptor over and above the standard two-point function $S_{2}$. Thus, $C_{2}$ has the ability to leapfrog past the usual approach of incorporating additional information via higher-order versions of $S_{2}$ (i.e, $S_{3}$, $S_{4}$, etc.) [29]. The present work clearly distinguishes the two-point probability and cluster functions $F_{2}$ and $K_{2}$, respectively, as highly sensitive descriptors of random media, since they are generalizations of $S_{2}$ and $C_{2}$ and hence contain even more information than the combination of $S_{2}$ and $C_{2}$.

The high information content of $F_{2}$ and $K_{2}$ suggests that it would be profitable to explore their use to characterize the structure and physical properties of not only random media, but molecular systems. For example, these functions might provide sensitive structural signatures of glassy states of matter (an issue of current interest [30]) and improved rigorous upper and lower bounds on the effective properties of a heterogeneous material [1]. These and related issues are beyond the scope of the present work, and hence represent interesting future research topics.

As noted earlier, two-phase media with percolating phases are difficult to target directly with $C_{2}$, which becomes long-ranged at the percolation threshold. However, preliminary reconstruction results for such cases obtained by applying dilation and erosion processes to generate a nonpercolating target medium, which is then reconstructed using the generalized $S_{2}$ and $C_{2}$, are promising and will be discussed further in future work.

Although we have focused in this paper on reconstructions of two-dimensional heterogeneous media in order to justify our methods, previous work on reconstructions using $S_{2}$ and $C_{2}$ by Jiao, Stillinger, and Torquato [15] suggests that our methods can be easily extended to higher dimensions. One interesting three-dimensional application of our work is the reconstruction of biconnected media, in which both phases percolate simultaneously. This behavior, though difficult to realize in two dimensions, is more common in three dimensions 1, 10], with important implications for the effective properties of a heterogeneous material [1]. 


\section{ACKNOWLEDGMENTS}

This work was supported by the Office of Basic Energy Sciences, Divsions of Materials Sciences and Engineering, under Award DE-FG02-04-ER46108.

[1] S. Torquato, Random Heterogeneous Materials: Microstructure and Macroscopic Properties, (Springer, New York, 2002).

[2] M. Sahimi, Heterogeneous Materials (Springer, New York, 2003); H. Hamzehpour, M. Reza Rasaei, and M. Sahimi, Phys. Rev. E 75, 056311 (2007).

[3] T. I. Zohdi, Mech. Mater. 38, 969 (2006).

[4] A. Mejdoubi and C. Brosseau, J. Appl. Phys. 101, 084109 (2007).

[5] E. J. Garboczi and D. P. Bentz, J. Adv. Cement-Based Mater. 8, 77 (1998).

[6] P. J. E. Peebles, Principles of Cosmology, (Princeton University Press, Princeton, 1993).

[7] K-G. Lee and D. N. Spergel, Astrophys. J. 734, 21 (2011).

[8] L. J. Gibson and M. F. Ashby, Cellular Solids: Structure and Properties, 2nd ed. (Cambridge UP, Cambridge, 1997).

[9] J. L. Gevertz and S. Torquato, PLoS Comput. Bio., 4, e1000152 (2008).

[10] A. P. Roberts and M. Teubner, Phys. Rev. E 51, 4141 (1995).

[11] C. L. Y. Yeong and S. Torquato, Phys. Rev. E 57, 495 (1998); ibid., Phys. Rev. E 58, 224 (1998).

[12] Y. Jiao, F. H. Stillinger, and S. Torquato, Phys. Rev. E 81, 011105 (2010); ibid., Phys. Rev. E. 82, 011106 (2010). It has been rigorously shown in these papers that specifying only a finite number of correlation functions defines a class of microstructures with degenerate statistics.

[13] H. Kumar, C. L. Briant, and W. A. Curtin, Mech. Mater. 38, 818 (2006).

[14] M. G. Rozman and M. Utz, Phys. Rev. Lett. 89, 135501 (2002).

[15] Y. Jiao, F. H. Stillinger, and S. Torquato, Proc. Nat. Acad. Sci. (USA) 106, 17634 (2009).

[16] D. T. Fullwood, S. R. Niezgoda, B. L. Adams, and S. R. Kalidindi, Prog. Mater. Sci. 55, 477 (2010).

[17] S. Torquato, J. Stat. Phys. 45, 843 (1986).

[18] P. Debye and A. M. Bueche, J. Appl. Phys. 20, 518 (1949). 
[19] M. D. Rintoul, S. Torquato, C. Yeong, D. T. Keane, S. Erramilli, Y. N. Jun, D. Dabbs and I. A. Aksay, Phys. Rev. E 54, 2663 (1996).

[20] S. Torquato, J. D. Beasley, and Y. C. Chiew, J. Chem. Phys. 88, 6540 (1988).

[21] J. Quintanilla and S. Torquato, Adv. Appl. Prob. 29, 327 (1997).

[22] L. V. Gibianky and S. Torquato, J. Mech. Phys. Solids 44, 233 (1996).

[23] S. Torquato, Phys. Rev. E 82, 056109 (2010).

[24] C. E. Zachary and S. Torquato, Phys. Rev. E 83, 051133 (2011).

[25] Y. Jiao, F. H. Stillinger, and S. Torquato, Phys. Rev. E 77, 031135 (2008).

[26] J. Kim, H. S. Kim, N. Lee, T. Kim, H. Kim, T. Yu, I. C. Song, W. K. Moon, and T. Hyeon, Angew. Chem. Int. Ed. 47, 8438 (2008).

[27] V. Springel, et. al., Nature 435, 629 (2005).

[28] C. H. Arns, M. A. Knackstedt, and K. Mecke, J. Microscopy 240, 181 (2010).

[29] S. Torquato and G. Stell, J. Chem. Phys. 78, 3262 (1983).

[30] V. Lubchenko and P. G. Wolynes, Ann. Rev. Phys. Chem. 58, 235 (2006); L. Berthier, G. Biroli, J-P. Bouchaud, J. W. Kob, K. Miyazaki, D. R. Reichman, J. Chem. Phys. 126, 184503 (2007); D. Chandler and J. P. Garrahan, Annu. Rev. Phys. Chem. 61, 191 (2010); L. Berthier and G. Tarjus, J. Chem. Phys. 134, 214503 (2011). 